\title{
Optimal Control Problem for the Weak Nonlinear Equation of Thin Plate With Control at the Coefficient of Lowest Term
}

\author{
Khayala I. Seyfullaeva \\ Correspondence: Sumgait State University, Sumgait, 43rd block, AZ5008, Azerbaijan
}

Received: March 1, 2020 Accepted: April 16, 2020 Online Published: April 26, 2020

doi:10.5539/jmr.v12n3p31 URL: https://doi.org/10.5539/jmr.v12n3p31

\begin{abstract}
The paper deals with an inverse problem of determining the right-hand side of the linear equation of oscillations of thin plates. The problem is reduced to the optimal control problem. Differentiability of the functional is studied. Necessary condition of optimality is derived.
\end{abstract}

Keywords: thin plate, optimal control, adjoint problem, optimality conditions

\section{Introduction}

It is known that the oscillations of thin plates are described by the differential equations with partial derivatives of the fourth order. For more detail, the reader is referred to (Komkov, 1975) and (Arman, 1977). Therefore, the study of optimal control problems for the equation of the thin plate is of great theoretical and practical importance, see (Kabanikhin, 2009). One of the approaches for solving the inverse problems is the optimization method. The essence of this method lies in the fact that the inverse problem is reduced to the optimal control problem and this new problem is investigated by the methods of optimal control theory.

The study of such problems started from the end of the twentieth century and is now intensively studied by many authors. See for examples: (Kabanikhin, 2009), (Alifanov, Artyukhin, \& Rumyantsev, 1988), (Sadek, Adali, Sloss, \& Bruchjr, 1992), (Blanton \& Sadek, 1994), (Tong, Williams, \& Agrawal, 1998), (X. Zhang \& J. Zhang, 1998), (Deineka, 2006) and (Bouchitte \& Fragala, 2007).

\section{Formulation of the Problem}

Our needs to find the pair of functions $(u, v) \in U \times U_{a d}$ from the relations

$$
\begin{aligned}
& \rho \frac{\partial^{2} u}{\partial t^{2}}+\Delta(D \Delta u)+(1-v)\left(2 \frac{\partial^{2} D}{\partial x \partial y} \frac{\partial^{2} u}{\partial x \partial y}-\frac{\partial^{2} D}{\partial x^{2}} \frac{\partial^{2} u}{\partial y^{2}}-\frac{\partial^{2} D}{\partial y^{2}} \frac{\partial^{2} u}{\partial x^{2}}\right)+v(x, y) u+|u| u= \\
& =f(x, y, t),(x, y, t) \in Q \text {, } \\
& u(x, y, 0)={ }_{0}(x, y), \frac{\partial u(x, y, 0)}{\partial t}={ }_{1}(x, y),(x, y) \in \Omega, \\
& u(0, y, t)=0, \frac{\partial u(0, y, t)}{\partial x}=0, u(x, 0, t)=0, \frac{\partial u(x, 0, t)}{\partial y}=0, \quad 0 \leq x \leq a, \quad 0 \leq t \leq T, \\
& u(a, y, t)=0, \frac{\partial u(a, y, t)}{\partial x}=0, u(x, b, t)=0, \frac{\partial u(x, b, t)}{\partial y}=0, \quad 0 \leq y \leq b, \quad 0 \leq t \leq T \\
& \int_{0}^{T} K(x, y, t) u(x, y, t) d t=g(x, y),
\end{aligned}
$$

where $(x, y) \in \Omega=\{(x, y): 0<x<a, 0<y<b\}, t \in(0, T), Q=\Omega \times(0, T), \rho(x, y)$ is a density of the mass at the point $(x, y), h(x, y)$ is the heath thickness of the plate in the point $(x, y), u(x, y, t)$ - is deflection of the plate in 
the point $(x, y)$ at the moment $t, \Delta$ is Laplace operator with respect to $x, y, D=\frac{E h^{3}}{12\left(1-v^{2}\right)}$ - cylindrical rigidity,

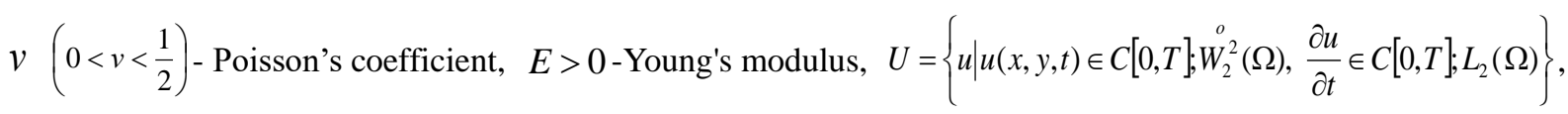
$U_{a d}=\left\{v(x, y) \in L_{2}(\Omega): \mu_{0} \leq v(x, y) \leq \mu_{1}\right.$ a.e. on $\left.\Omega\right\} \quad, \quad{ }_{0}(x, y) \in \stackrel{o}{W_{2}^{2}}(\Omega) \quad, \quad{ }_{1}(x, y) \in L_{2}(\Omega) \quad, \quad K(x, y, t) \in L_{\infty}(Q) \quad$, $g(x, y) \in L_{2}(\Omega)$ are given functions, $h(x, y)$ is a sufficiently smooth given function, $a, b, \mu_{0}, \mu_{1}, T$ are given positive numbers.

As a generalized solution of the problem (1)-(3) for each function $v(x, y)$ from $L_{2}(\Omega)$, we consider the function $u(x, y, t) \in U$ such that for any $\forall \eta(x, y, t) \in U, \eta(x, y, T)=0$ the integral identity

$$
\begin{gathered}
\int_{Q}\left[-\rho \frac{\partial u}{\partial t} \cdot \frac{\partial \eta}{\partial t}+D \Delta u \Delta \eta+(1-v)\left(2 \frac{\partial^{2} D}{\partial x \partial y} \frac{\partial^{2} u}{\partial x \partial y}-\frac{\partial^{2} D}{\partial x^{2}} \frac{\partial^{2} u}{\partial y^{2}}-\frac{\partial^{2} D}{\partial y^{2}} \frac{\partial^{2} u}{\partial x^{2}}\right) \eta\right] d x d y d t- \\
-\int_{\Omega} \rho_{1}(x, y) \eta(x, y, 0) d x d y+\int_{Q} v u \eta d x d y d t+\int_{Q}|u| u \eta d x d y d t=\int_{Q} f(x, y, t) \eta d x d y d t .
\end{gathered}
$$

is fulfilled.

This problem we reduce to the following optimal control problem: to find the minimum of the functional

$$
J_{0}(v)=\frac{1}{2} \iint_{\Omega}^{T}\left[\int_{0}^{T} K(x, y, t) u(x, y, t ; v) d t-g(x, y)\right]^{2} d x d y,
$$

subject to (1)-(3). The function $v(x, y)$ is called a control. By $u=u(x, y, t ; v)$ we denote the generalized solution of the problem (1)-(3) corresponding to the control $v(x, y)$.

We regularize the problem (1)-(3), (6) by the following way: instead of the functional (6) consider the next one

$$
J_{\alpha}(v)=J_{0}(v)+\frac{\alpha}{2} \int_{\Omega} v^{2}(x, y) d x d y,
$$

where $\alpha>0$ is a positive number.

Note that by any fixed control $v(x, y)$ boundary problem (1)-(3) has unique generalized solution from $U$ (Quliyev \& Seyfullayeva, 2013).

In the considered problem all condition for existence of optimal control are fulfilled (Lions, 1972). Therefore the new optimal control problem (1)-(3), (7) also has unique solution.

\section{Existence of the Optimal Control}

Theorem 1. Under the imposed conditions on the problem data, there exists an optimal control in problem (1)-(3), (7).

Proof. Let's $\left\{v_{n}\right\} \in U_{a d}$ be a minimizing sequence, i.e.

$$
\lim _{n \rightarrow \infty} J\left(v_{n}\right)=\inf _{v \in U_{a d}} J(v) .
$$

It is obvious that

$$
\left\|v_{n}\right\|_{L_{2}(\Omega)} \leq \text { const }
$$

Therefore by (8), for solutions of problem (1)-(3) corresponding to $v_{n}$, we obtain the estimation

$$
\left\|u_{n}\right\|_{W_{2}^{2}(\Omega)}^{o}+\left\|\frac{\partial u_{n}}{\partial t}\right\|_{L_{2}(\Omega)} \leq \text { const }, \forall t \in[0, T] .
$$

By (8) and (9), property of weak compactness in the Hilbert spaces and imbedding theorem, it is possible to consider, that as $n \rightarrow \infty$ 


$$
\begin{gathered}
v_{n} \rightarrow v_{0} \text { weakly in } L_{2}(\Omega), \\
u_{n} \rightarrow u_{0}, \frac{\partial u_{n}}{\partial x} \rightarrow \frac{\partial u_{0}}{\partial x}, \frac{\partial u_{n}}{\partial y} \rightarrow \frac{\partial u_{0}}{\partial y} \text { strongly in } L_{2}(Q), \\
\frac{\partial^{2} u_{n}}{\partial x^{2}} \rightarrow \frac{\partial^{2} u_{0}}{\partial x^{2}}, \frac{\partial^{2} u_{n}}{\partial x \partial y} \rightarrow \frac{\partial^{2} u_{0}}{\partial x \partial y}, \frac{\partial^{2} u_{n}}{\partial y^{2}} \rightarrow \frac{\partial^{2} u_{0}}{\partial y^{2}} \text { weakly in } L_{2}(Q) .
\end{gathered}
$$

Take into account last relations in the definition of the generalized solution for the problem (1)-(3), by $v=v_{n}, u=u_{n}$, passing to limit as $n \rightarrow \infty$, we have

$$
\begin{gathered}
\int_{Q}\left[-\rho \frac{\partial u_{0}}{\partial t} \cdot \frac{\partial \eta}{\partial t}+D_{0} \Delta u_{0} \Delta \eta+(1-v)\left(2 \frac{\partial^{2} D_{0}}{\partial x \partial y} \frac{\partial^{2} u}{\partial x \partial y}-\frac{\partial^{2} D_{0}}{\partial x^{2}} \frac{\partial^{2} u}{\partial y^{2}}-\frac{\partial^{2} D_{0}}{\partial y^{2}} \frac{\partial^{2} u}{\partial x^{2}}\right) \eta\right] d x d y d t- \\
-\int_{\Omega} \rho_{1}(x, y) \eta(x, y, 0) d x d y+\int_{Q} v_{0} u_{0} \eta d x d y d t+\int_{Q}\left|u_{0}\right| u_{0} \eta d x d y d t=\int_{Q} f(x, y, t) \eta d x d y d t .
\end{gathered}
$$

Therefore,

$$
\lim _{n \rightarrow \infty} J\left(v_{n}\right)=\inf _{v \in U_{a d}} J(v)=J\left(v_{0}\right) .
$$

It shows that $v_{0}(x, y)$ provides the minimum to functional (7), i.e. is an optimal control.

The completes the proof.

\section{Differentiability of the Functional (7) and Necessary and Sufficient Optimality Conditions}

Let us introduce the adjoint problem to (1)-(3), (7) problem for the given control $v(x, y, t) \in L_{2}(Q)$ :

$$
\begin{gathered}
\rho \frac{\partial^{2} \psi}{\partial t^{2}}+\Delta(D \Delta \psi)+(1-v)\left[2 \frac{\partial^{2}}{\partial x \partial y}\left(\frac{\partial^{2} D}{\partial x \partial y} \psi\right)-\frac{\partial^{2}}{\partial x^{2}}\left(\frac{\partial^{2} D}{\partial y^{2}} \psi\right)-\frac{\partial^{2}}{\partial y^{2}}\left(\frac{\partial^{2} D}{\partial x^{2}} \psi\right)\right]+v \psi+2|u| \psi= \\
=-K(x, y, t)\left[\int_{0}^{T} K(x, y, t) u(x, y, t) d t-g(x, y)\right],(x, y, t) \in Q, \\
\psi(x, y, T)=0, \rho \frac{\partial \psi(x, y, T)}{\partial t}=0, \quad(x, y) \in \Omega, \\
\psi(0, y, t)=\psi(a, y, t)=0, \frac{\partial \psi(0, y, t)}{\partial x}=\frac{\partial \psi(a, y, t)}{\partial x}=0, \quad 0 \leq x \leq a, \quad 0 \leq t \leq T, \\
\psi(x, 0, t)=\psi(x, b, t)=0, \frac{\partial \psi(x, 0, t)}{\partial y}=\frac{\partial \psi(x, b, t)}{\partial y}=0, \quad 0 \leq y \leq b, \quad 0 \leq t \leq T .
\end{gathered}
$$

From the conditions imposed on the data of the problem (1)-(3), (7) follows that this adjoint problem has unique generalized solution from the space $W_{2}^{2,1}(Q)$ (Quliyev \& Seyfullayeva, 2013).

To derive the necessary conditions for optimality in the considered problem we take two arbitrary admissible controls $v(x, y)$ and $v(x, y)+\delta v(x, y)$. The corresponding solutions of problem (1)-(3) are denoted by $u(x, y, t ; v)$ and $u(x, y, t ; v+\delta v) \equiv u(x, y, t ; v)+\delta u(x, y, t)$. Then $\delta u(x, y, t)=u(x, y, t ; v+\delta v)-u(x, y, t ; v)$ is a solution of the boundary value problem

$$
\begin{gathered}
\rho \frac{\partial^{2}(\delta u)}{\partial t^{2}}+\Delta(D \Delta(\delta u))+(1-v)\left[2 \frac{\partial^{2} D}{\partial x \partial y} \frac{\partial^{2}(\delta u)}{\partial x \partial y}-\frac{\partial^{2} D}{\partial x^{2}} \frac{\partial^{2}(\delta u)}{\partial y^{2}}-\frac{\partial^{2} D}{\partial y^{2}} \frac{\partial^{2}(\delta u)}{\partial x^{2}}\right]+ \\
+(v+\delta v) \delta u+2|u+\theta \delta u| \delta u=-u \delta v \\
\delta u(x, y, 0)=0, \frac{\partial(\delta u(x, y, 0))}{\partial t}=0,
\end{gathered}
$$




$$
\begin{aligned}
& \delta u(0, y, t)=\delta u(a, y, t)=0, \frac{\partial(\delta u(0, y, t))}{\partial x}=\frac{\partial(\delta u(a, y, t))}{\partial x}=0, \\
& \delta u(x, 0, t)=\delta u(x, b, t)=0, \frac{\partial(\delta u(x, 0, t))}{\partial y}=\frac{\partial(\delta u(x, b, t))}{\partial y}=0 .
\end{aligned}
$$

Let's show that

$$
\|\delta u\|_{W_{2}^{2}(\Omega)}^{o}+\left\|\frac{\partial \delta u}{\partial t}\right\|_{L_{2}(\Omega)} \leq C\|\delta v\|_{L_{2}(\Omega)}, \quad \forall t \in[0, T] .
$$

For this purpose, we use Faedo-Galerkin's method. Take the basis $\left\{\omega_{i}(x, y)\right\}_{i=1}^{\infty}$ from $\dot{W}_{2}^{2}(\Omega)$, where the system $\left\{\omega_{i}(x, y)\right\}_{i=1}^{\infty}$ is orthonormal in $L_{2}(\Omega)$ and the approximate solution for the problem (14)-(16) search in the form

$$
\delta u^{N}(x, y, t)=\sum_{i=1}^{N} c_{i}^{N}(t) \omega_{i}(x, y)
$$

from the equalities

$$
\begin{gathered}
\int_{\Omega} \rho \frac{\partial^{2} \delta u^{N}}{\partial t^{2}} \omega_{j}(x, y) d x d y+\int_{\Omega} D \Delta \delta u^{N} \Delta \omega_{j}(x, y) d x d y+ \\
+(1-v) \int_{\Omega}\left(2 \frac{\partial^{2} D}{\partial x \partial y} \frac{\partial^{2} \delta u^{N}}{\partial x \partial y}-\frac{\partial^{2} D}{\partial x^{2}} \frac{\partial^{2} \delta u^{N}}{\partial y^{2}}-\frac{\partial^{2} D}{\partial y^{2}} \frac{\partial^{2} \delta u^{N}}{\partial x^{2}}\right) \omega_{j}(x, y) d x d y+ \\
+\int_{Q}(v+\delta v) \delta u \omega_{j}(x, y) d x d y d t+2 \int_{\Omega}\left|u+\theta \delta u^{N}\right| \delta u^{N} \omega_{j}(x, y) d x d y= \\
=-\int_{\Omega} u \delta v \omega_{j}(x, y) d x d y+\int_{\Omega} f(x, y, t) \omega_{j}(x, y) d x d y, \quad 1 \leq j \leq N, \\
c_{i}^{N}(0)=0,\left.\frac{d}{d t} c_{i}^{N}(t)\right|_{t=0}=0 .
\end{gathered}
$$

Multiplying both sides of (17) by $\frac{d}{d t} c_{j}^{N}(t)$ and sum over $j$ from 1 to $N$, we get

$$
\begin{gathered}
\int_{\Omega} \rho \frac{\partial^{2} \delta u^{N}}{\partial t^{2}} \frac{\partial \delta u^{N}}{\partial t} d x d y+\int_{\Omega} D \Delta \delta u^{N} \Delta \frac{\partial \delta u^{N}}{\partial t} d x d y+ \\
+(1-v) \int_{\Omega}\left(2 \frac{\partial^{2} D}{\partial x \partial y} \frac{\partial^{2} \delta u^{N}}{\partial x \partial y}-\frac{\partial^{2} D}{\partial x^{2}} \frac{\partial^{2} \delta u^{N}}{\partial y^{2}}-\frac{\partial^{2} D}{\partial y^{2}} \frac{\partial^{2} \delta u^{N}}{\partial x^{2}}\right) \frac{\partial \delta u^{N}}{\partial t} d x d y+ \\
+\int_{Q}(v+\delta v) \delta u \frac{\partial \delta u^{N}}{\partial t} d x d y d t+2 \int_{\Omega}\left|u+\theta \delta u^{N}\right| \delta u^{N} \frac{\partial \delta u^{N}}{\partial t} d x d y= \\
=-\int_{Q} u \delta v \frac{\partial \delta u^{N}}{\partial t} d x d y d t+\int_{\Omega} f(x, y, t) \frac{\partial \delta u^{N}}{\partial t} d x d y
\end{gathered}
$$

It gives that

$$
\frac{1}{2} \frac{d}{d t} \int_{\Omega}\left[\rho\left(\frac{\partial \delta u^{N}}{\partial t}\right)^{2}+D\left(\Delta \delta u^{N}\right)^{2}\right] d x d y=
$$




$$
\begin{aligned}
=-(1-v) \int_{\Omega}\left(2 \frac{\partial^{2} D}{\partial x \partial y} \frac{\partial^{2} \delta u^{N}}{\partial x \partial y}-\frac{\partial^{2} D}{\partial x^{2}} \frac{\partial^{2} \delta u^{N}}{\partial y^{2}}-\frac{\partial^{2} D}{\partial y^{2}} \frac{\partial^{2} \delta u^{N}}{\partial x^{2}}\right) \frac{\partial \delta u^{N}}{\partial t} d x d y- \\
-\int_{Q}(v+\delta v) \delta u^{N} \frac{\partial \delta u^{N}}{\partial t} d x d y d t-2 \int_{\Omega}\left|u+\theta \delta u^{N}\right| \delta u^{N} \frac{\partial \delta u^{N}}{\partial t} d x d y- \\
-\int_{Q} u \delta v \frac{\partial \delta u^{N}}{\partial t} d x d y d t+\int_{\Omega} f(x, y, t) \frac{\partial \delta u^{N}}{\partial t} d x d y .
\end{aligned}
$$

If to integrate this equality over $t$ by the imposed conditions, we get

$$
\begin{gathered}
\int_{\Omega}\left[\left(\frac{\partial \delta u^{N}(x, y, t)}{\partial t}\right)^{2}+\left(\Delta \delta u^{N}(x, y, t)\right)^{2}\right] d x d y \leq \\
\leq 2 \int_{0}^{t} \int_{\Omega}|u| \delta u^{N}\left|\frac{\partial \delta u^{N}(x, y, t)}{\partial t}\right| d x d y d s+ \\
+2 \int_{0}^{t} \int_{\Omega}\left|\delta u^{N}\right|^{2}\left|\frac{\partial \delta u^{N}(x, y, t)}{\partial t}\right| d x d y d s+\int_{0}^{t} \int|u||\delta v| \frac{\partial \delta u^{N}(x, y, t)}{\partial t} \mid d x d y d s \leq \\
\leq 2 \int_{0}^{t} \int_{\Omega}|u|^{2}\left|\delta u^{N}\right|^{2} d x d y d s+2 \int_{0}^{t} \int_{\Omega}\left|\frac{\partial \delta u^{N}(x, y, t)}{\partial t}\right|^{2} d x d y d s+\int_{0 \Omega}^{t} \int_{\Omega}\left|\delta u^{N}\right|^{4} d x d y d s+ \\
+\int_{0 \Omega}^{t} \int_{\Omega}|u|^{2}|\delta v|^{2} d x d y d s+\int_{0}^{t} \int_{\Omega}\left|\frac{\partial \delta u^{N}(x, y, t)}{\partial t}\right|^{2} d x d y d s \leq \\
\leq C \int_{0}^{t} \int_{\Omega}\left[\left(\delta u^{N}(x, y, s)\right)^{2}+\left(\frac{\partial \delta u^{N}(x, y, s)}{\partial t}\right)^{2}+\left(\frac{\partial \delta u^{N}(x, y, s)}{\partial x}\right)^{2}+\left(\frac{\partial \delta u^{N}(x, y, s)}{\partial y}\right)^{2}+\right. \\
\left.+\left(\frac{\partial^{2} \delta u^{N}(x, y, s)}{\partial x^{2}}\right)^{2}+\left(\frac{\partial^{2} \delta u^{N}(x, y, s)}{\partial x \partial y}\right)^{2}+\left(\frac{\partial^{2} \delta u^{N}(x, y, s)}{\partial y^{2}}\right)^{2}\right] d x d y d s+C|| \delta v \|_{L_{2}(\Omega)}^{2}, \forall t \in[0, T],
\end{gathered}
$$

where $C$ the constant independent on the estimating quantities and admissible controls.

Due to equivalency of the norms in $W_{2}^{2}(\Omega)$ we obtain

$$
\begin{gathered}
\int_{\Omega}\left[\left(\delta u^{N}(x, y, t)\right)^{2}+\left(\frac{\partial \delta u^{N}(x, y, t)}{\partial t}\right)^{2}+\left(\frac{\partial \delta u^{N}(x, y, t)}{\partial x}\right)^{2}+\left(\frac{\partial \delta u^{N}(x, y, t)}{\partial y}\right)^{2}+\left(\Delta \delta u^{N}(x, y, t)\right)^{2}\right] d x d y \leq \\
\leq C \int_{0}^{t} \int_{\Omega}\left[\left(\frac{\partial \delta u^{N}(x, y, s)}{\partial t}\right)^{2}+\left(\frac{\partial \delta u^{N}(x, y, s)}{\partial x}\right)^{2}+\left(\frac{\partial \delta u^{N}(x, y, s)}{\partial y}\right)^{2}+\right. \\
\left.+\left(\frac{\partial^{2} \delta u^{N}(x, y, s)}{\partial x^{2}}\right)^{2}+\left(\frac{\partial^{2} \delta u^{N}(x, y, s)}{\partial x \partial y}\right)^{2}+\left(\frac{\partial^{2} \delta u^{N}(x, y, s)}{\partial y^{2}}\right)^{2}\right] d x d y d s+C\|\delta v\|_{L_{2}(\Omega)}^{2} .
\end{gathered}
$$

By virtue of well-known inequality (Ladijenskaya, 1973)

$$
\int_{\Omega}\left[\left(\frac{\partial^{2} \delta u^{N}}{\partial x^{2}}\right)^{2}+\left(\frac{\partial^{2} \delta u^{N}}{\partial x \partial y}\right)^{2}+\left(\frac{\partial^{2} \delta u^{N}}{\partial y^{2}}\right)^{2}\right] d x d y \leq \int_{\Omega}\left[\frac{\partial^{2} \delta u^{N}}{\partial x^{2}}+\frac{\partial^{2} \delta u^{N}}{\partial y^{2}}\right]^{2} d x d y
$$

and by (18), we have 


$$
\begin{gathered}
\int_{\Omega}\left[\left(\delta u^{N}(x, y, t)\right)^{2}+\left(\frac{\partial \delta u^{N}(x, y, t)}{\partial t}\right)^{2}+\left(\frac{\partial \delta u^{N}(x, y, t)}{\partial x}\right)^{2}+\left(\frac{\partial \delta u^{N}(x, y, t)}{\partial y}\right)^{2}+\right. \\
\left.+\left(\frac{\partial^{2} \delta u^{N}(x, y, t)}{\partial x^{2}}\right)^{2}+\left(\frac{\partial^{2} \delta u^{N}(x, y, t)}{\partial x \partial y}\right)^{2}+\left(\frac{\partial^{2} \delta u^{N}(x, y, t)}{\partial y^{2}}\right)^{2}\right] d x d y \leq \\
\leq C \int_{0 \Omega}^{t} \int\left[\left(\delta u^{N}(x, y, s)\right)^{2}+\left(\frac{\partial \delta u^{N}(x, y, s)}{\partial t}\right)^{2}+\left(\frac{\partial \delta u^{N}(x, y, s)}{\partial x}\right)^{2}+\left(\frac{\partial \delta u^{N}(x, y, s)}{\partial y}\right)^{2}+\right. \\
\left.+\left(\frac{\partial^{2} \delta u^{N}(x, y, s)}{\partial x^{2}}\right)^{2}+\left(\frac{\partial^{2} \delta u^{N}(x, y, s)}{\partial x \partial y}\right)^{2}+\left(\frac{\partial^{2} \delta u^{N}(x, y, s)}{\partial y^{2}}\right)^{2}\right] d x d y d s+C\|\delta v\|_{L_{2}(\Omega)}^{2}
\end{gathered}
$$

Application of the Gronwall's lemma leads to

$$
\begin{gathered}
\int_{\Omega}\left[\left(\delta u^{N}(x, y, t)\right)^{2}+\left(\frac{\partial \delta u^{N}(x, y, t)}{\partial t}\right)^{2}+\left(\frac{\partial \delta u^{N}(x, y, t)}{\partial x}\right)^{2}+\left(\frac{\partial \delta u^{N}(x, y, t)}{\partial y}\right)^{2}+\right. \\
\left.+\left(\frac{\partial^{2} \delta u^{N}(x, y, t)}{\partial x^{2}}\right)^{2}+\left(\frac{\partial^{2} \delta u^{N}(x, y, t)}{\partial x \partial y}\right)^{2}+\left(\frac{\partial^{2} \delta u^{N}(x, y, t)}{\partial y^{2}}\right)^{2}\right] d x d y \leq C\|\delta\|_{L_{2}(\Omega)}^{2}, \forall t \in[0, T]
\end{gathered}
$$

From this integrating over $t$ in (19), we get

$$
\left\|\delta u^{N}\right\|_{W_{2}^{2}(\Omega)}^{o}+\left\|\frac{\partial \delta u^{N}}{\partial t}\right\|_{L_{2}(\Omega)} \leq C\|\delta v\|_{L_{2}(\Omega)}^{2}, \forall t \in[0, T] .
$$

As follows from this inequality from the sequence $\left\{\delta u^{N}(x, y, t)\right\}$ one can chose a subsequence (which is also denoted by $\left.\left\{\delta u^{N}(x, y, t)\right\}\right)$ that converges weakly in $U$ to some function $\delta u(x, y, t)$ by $N \rightarrow \infty$.

Thus, by the weak lower semi-continuity of the norm in the Banach space, (20) implies estimate (16).

Theorem 2. Let's all conditions of the Theorem 1 be satisfied. Then functional (7) is continuously Frechet differentiable on $U_{a d}$ and its differential in the point $\forall v \in U_{a d}$ at the increment $\delta v \in L_{2}(\Omega), v+\delta v \in U_{a d}$ is defined by the expression

$$
\left\langle J_{\alpha}^{\prime}(v), \delta v\right\rangle=\int_{\Omega}\left[\alpha v(x, y)+\int_{0}^{T} u(x, y, t) \psi(x, y, t) d t\right] \delta v d x d y .
$$

Proof. Let's calculate the increment of the functional $J_{\alpha}(v)$ :

$$
\begin{gathered}
\Delta J_{\alpha}(v)=J_{\alpha}(v+\delta v)-J_{\alpha}(v)= \\
=\frac{1}{2} \int_{\Omega}\left(\int_{0}^{T} K(u+\delta u) d t-g(x, y)\right)^{2} d x d y-\frac{1}{2} \int_{\Omega}\left(\int_{0}^{T} K u d t-g(x, y)\right)^{2} d x d y+\frac{\alpha}{2} \int_{\Omega}\left[(v+\delta v)^{2}-v^{2}\right] d t= \\
=\int_{\Omega}\left[\left(\int_{0}^{T} K u d t-g(x, y)\right)_{0}^{T} K \delta u d t\right] d x d y+\frac{1}{2} \int_{\Omega}\left(\int_{0}^{T} K \delta u d t\right)^{2} d x d y+\alpha \int_{\Omega} v \delta v d t+\frac{\alpha}{2} \int_{\Omega}(\delta v)^{2} d t, \\
\Delta J_{\alpha}(v)=\int_{\Omega}\left(\int_{0}^{T} K u d t-g(x, y)\right)_{0}^{T} K \delta u d x d y d t+\alpha \int_{\Omega} v \delta v d t+R_{1},
\end{gathered}
$$

where

$$
R_{1}=\frac{1}{2} \int_{\Omega}\left(\int_{0}^{T} K \delta u d t\right)^{2} d x d y+\frac{\alpha}{2} \int_{\Omega}(\delta v)^{2} d t
$$

is the remainder term.

Since $\delta u$ is a generalized solution of the problem (13)-(16), for arbitrary function $\eta(x, y, t) \in U, \eta(x, y, T)=0$, 


$$
\begin{aligned}
& \eta(0, y, t)=0, \frac{\partial \eta(0, y, t)}{\partial x}=0, \eta(x, 0, t)=0, \frac{\partial \eta(x, 0, t)}{\partial y}=0 \\
& \eta(a, y, t)=0, \frac{\partial \eta(a, y, t)}{\partial x}=0, \eta(x, b, t)=0, \frac{\partial \eta(x, b, t)}{\partial y}=0 .
\end{aligned}
$$

is valid integral identity

$$
\begin{aligned}
\int_{Q}\left\{-\rho \frac{\partial(\delta u)}{\partial t} \frac{\partial \eta}{\partial t}+\right. & D \Delta(\delta u) \Delta \eta+(1-v)\left[2 \frac{\partial^{2} D}{\partial x \partial y} \frac{\partial^{2}(\delta u)}{\partial x \partial y}-\frac{\partial^{2} D}{\partial x^{2}} \frac{\partial^{2}(\delta u)}{\partial y^{2}}-\frac{\partial^{2} D}{\partial y^{2}} \frac{\partial^{2}(\delta u)}{\partial x^{2}}\right] \eta+ \\
& +(v+\delta v) \delta u \eta+2|u+\theta \delta u| \delta u \eta\} d x d y d t=-\int_{Q} u \delta v \eta d x d y d t .
\end{aligned}
$$

Similarly, since $\psi(x, y, t)$ is a solution of the problem (10)-(12), for any function $\chi(x, y, t) \in U, \chi(x, y, 0)=0$,

$$
\begin{aligned}
& \chi(0, y, t)=0, \frac{\partial \chi(0, y, t)}{\partial x}=0, \chi(x, 0, t)=0, \frac{\partial \chi(x, 0, t)}{\partial y}=0, \\
& \chi(a, y, t)=0, \frac{\partial \chi(a, y, t)}{\partial x}=0, \chi(x, b, t)=0, \frac{\partial \chi(x, b, t)}{\partial y}=0
\end{aligned}
$$

we have

$$
\begin{aligned}
& \int_{Q}\left\{-\rho \frac{\partial \psi}{\partial t} \frac{\partial \chi}{\partial t}+D \Delta \psi \Delta \chi+(1-v)\left[2 \frac{\partial^{2} D}{\partial x \partial y} \psi \frac{\partial^{2} \chi}{\partial x \partial y}-\frac{\partial^{2} D}{\partial x^{2}} \psi \frac{\partial^{2} \chi}{\partial y^{2}}-\frac{\partial^{2} D}{\partial y^{2}} \psi \frac{\partial^{2} \chi}{\partial x^{2}}\right]+v \psi \chi+2|u| \psi \chi\right\} d x d y d t= \\
& =-\int_{Q} K(x, y, t)\left[\int_{0}^{T} K(x, y, t) u(x, y, t) d t-g(x, y)\right] \chi d x d y d t . .
\end{aligned}
$$

Let $\psi(x, y, t)=\eta(x, y, t)$ and let $\delta u(x, y, t)=\chi(x, y, t)$ in (22) and (23), respectively. After subtracting (22) from (23), we obtain

$$
\begin{gathered}
\int_{\Omega}\left(\int_{0}^{T} K u d t-g(x, y)\right)_{0}^{T} \int_{0}^{T} K \delta u d x d y d t=\int_{Q} u(x, y, t) \delta v \psi(x, y, t) d x d y d t+ \\
+\int_{Q} \psi \delta u \delta v d x d y d t+\int_{Q} 2[|u+\theta \delta u|-|u|] \psi \delta u d x d y d t .
\end{gathered}
$$

Then from (21) and (24) follows

$$
\Delta J_{\alpha}(v)=\int_{\Omega}\left[\alpha v(x, y)+\int_{0}^{T} u(x, y, t) \psi(x, y, t) d t\right] \delta v d x d y+R,
$$

where

$$
\begin{gathered}
R=R_{1}+R_{2}, \\
R_{2}=\int_{Q} \psi \delta u \delta v d x d y d t+\int_{Q} 2[|u+\theta \delta u|-|u|] \psi \delta u d x d y d t .
\end{gathered}
$$

By (16), we obtain

$$
R \leq C\|\delta v\|_{L_{2}(\Omega)}^{2}
$$

Then from formula for increment of the functional (25) follows that differential of functional (7) is calculate by the formula

$$
\left\langle J_{\alpha}^{\prime}(v), \delta v\right\rangle=\int_{\Omega}\left[\alpha v(x, y)+\int_{0}^{T} u(x, y, t) \psi(x, y, t) d t\right] \delta v d x d y .
$$

Then as follows from (27) the gradient of the functional has a from

$$
\operatorname{grad} J(v)=u(x, y, t) \psi(x, y, t)+\alpha v(x, y) .
$$

Thus due to known theorem from (Vasilyev, 1981) in order to the control function $v_{*}(x, y)$ was optimal, it is necessary 
fulfillment of the inequality

$$
\int_{\Omega}\left[\alpha v_{*}(x, y)+\int_{0}^{T} u(x, y, t) \psi(x, y, t) d t\right]\left(v(x, y)-v_{*}(x, y)\right) d x d y \geq 0, \quad \forall v \in U_{a d} .
$$

Since the functional (7) is strongly convex in $U_{a d}$ and the problem (1)-(3) is linear, condition (28) is also sufficient for the optimality.

Thus the following theorem is proved.

Theorem 3. Let's the conditions of the Theorem 1 be satisfied. Then for the optimality of the control $v_{*} \in U_{a d}$ for problem (1)-(3), it is necessary the validity of the inequality

$$
\int_{\Omega}\left[\alpha v_{*}(x, y)+\int_{0}^{T} u(x, y, t) \psi(x, y, t) d t\right]\left(v(x, y)-v_{*}(x, y)\right) d x d y \geq 0
$$

for arbitrary $v=v(x, y) \in U_{a d}$, where $u_{*}(x, y, t)$ and $\psi_{*}(x, y, t)$ are solutions of problems (1)-(3), (10)-(12), respectively.

\section{References}

Alifanov, O. M., Artyukhin, E. A., \& Rumyantsev, S. V. (1988). Extremal methods for solving ill-posed problems and their applications to inverse heat transfer problems. Moscow, Nauka.

Arman, J. L. P. (1977). Applications of the optimal control theory for the distributed parameter systems to the problems of structural optimization. Moscow, Mir.

Blanton, M. L., \& Sadek, I. S. (1994). Optimal active pointwise of thin plates via state-control parametrization. International Journal of Systems Science, 25, 2001-2014. https://doi.org/ 10.1080/00207729408949329

Bouchitte, G., \& Fragala, I. (2007). Optimality conditions for Mass Design problems and applications to thin plates. Archive for Rational Mechanics and Analysis, 184, 257-284. https://doi.org/10.1007/s00205-006-0022-8

Deineka, V. S. (2006). Optimal control of the dynamic state of a thin compound plate. Cybernetics and Systems Analysis, 42, 151-175. https://doi.org/10.1007/s10559-006-0096-1

Kabanikhin, S. I. (2009). Inverse and Ill-posed problems. Novosibirsk, Russia.

Komkov, V. (1975). The theory of optimal control of damping vibrations of simple elastic systems. Moscow, Mir.

Ladijenskaya, O. A. (1973). Boundary value problems of mathematical physics. Moscow, Nauka.

Lions, J. L. (1972). Optimal control of the systems dscribed by the partial differential equations. Moscow, Mir.

Quliyev, H. F., \& Seyfullayeva, K. I. (2013). Optimal control problem for the equation of vibrations for thin plate. News of Baku University, Physico-mathematical sciences, 3, 64-73.

Sadek, S., Adali, S., Sloss, J. M., \& Bruchjr, J. C. (1992). Vibration damping of a thin plate by optimal open-and closed-loop control forces. Journal of the Franklin Institute, 329, 207-214. https://doi.org/10.1016/0016-0032(92)90029-G

Tong, D., Williams, R. L., \& Agrawal, S. K. (1998). Optimal shape control of composite thin plates with piezoelectric actuators. Journal of Intelligent Material Systems and Structures, 9, 458-467. https://doi.org/10.1177/1045389X9800900607

Vasilyev, F. P. (1981). Methods for solution of the extremal problems. Moscow, Nauka.

Zhang, X., \& Zhang, J. (1998). The hybrid control of vibration of thin plate with active constrained damping layer. Applied Mathematics and Mechanics, 19, 1119-1134. https://doi.org/10.1007/BF02456633

\section{Copyrights}

Copyright for this article is retained by the author(s), with first publication rights granted to the journal.

This is an open-access article distributed under the terms and conditions of the Creative Commons Attribution license (http://creativecommons.org/licenses/by/4.0/). 